\title{
ESTUDO DA DISPENSAÇÃO DE MEDICAMENTOS ANTI - RETROVIRAIS A PACIENTES INFECTADOS POR HIV NO SERVIÇO DE FARMÁCIA DO HC-UFG: PRIMEIRO PASSO NA IMPLANTAÇÃ̃O DA ATENÇÃO FARMACÊUTICA
}

\author{
Study of antiretroviral medicines dispensation to HIV infected patients by the pharmacy service \\ at HC- UFG: the first step to establishment of the pharmaceutical care
}

\author{
Iara A. L. Nogueira ${ }^{1 *}$; Aryane B. B. Leão²; Rúbia R. Bueno ${ }^{1,2}$; Amanda Q. Soares ${ }^{1}$; Renata \\ F. Carvalho ${ }^{1}$ \\ ${ }^{1}$ Seção de Farmácia, Hospital das Clínicas, Universidade Federal de Goiás, Goiânia, GO, Brasil. \\ ${ }^{2}$ Faculdade de Farmácia, Faculdades Objetivo, Goiânia, Go, Brasil
}

* Autor para correspondência email: iaranogueira@correios.net.br

Recebido em 11/04/2007- Aceito em 30/06/2007

\begin{abstract}
RESUMO: Neste trabalho avaliamos a dispensação de anti-retrovirais aos pacientes cadastrados no serviço de atendimento especial do Hospital das Clínicas /UFG, em particular observou-se o perfil farmacoepidemiológico, o esquema terapêutico, a retirada mensal de medicamentos, mudanças nos protocolos e, consequentemente a adesão dos pacientes ao tratamento. Todos os pacientes atendidos eram adultos, sendo $91(71,6 \%)$ do sexo masculino e $36(28,4 \%)$ do sexo feminino. A maioria ( $47,2 \%)$ estudaram somente até o ensino fundamental, eram casados ou tinham uma relação estável. Em relação a faixa etária mais prevalente foi a de 31-40 anos, mas em relação a mulheres a faixa etária mais prevalente foi a de $41-50$ anos. Em relação à aquisição mensal dos medicamentos na farmácia, 71 (55,9\%) pacientes receberam os anti-retrovirais regularmente, enquanto que 56 $(44,1 \%)$ pacientes em algum momento após o início do tratamento deixaram de adquirir os medicamentos mensalmente ou recebiam de forma irregular. Os dados obtidos indicam a necessidade de implantação de um serviço de atenção farmacêutica, para orientação dos pacientes e consequentemente melhora na aderência dos pacientes ao esquema terapêutico.
\end{abstract}

PALAVRAS-CHAVE: Aids; anti-retrovirais; dispensação.

ABSTRACT: In this work we evaluated the antiretroviral dispensation to patients enrolled at the special service pharmacy at the Hospital das Clínicas/UFG; in particular the pharmacoepidemiology, the therapeutic schedule, the monthly medicine acquisition, therapeutic schedule's changes, and adhesion were investigated. All the recorded patients were adult, $91(71.6 \%)$ male and 36(28.4\%) female. Most of them (47.2\%) had only elemental scholarship and also were married or with a stable relationship. The male group has age range of 31-41 years, whereas the female group had age range of 41-50 years. Regarding to the monthly medicine acquisition in the pharmacy service, $71(55.9 \%)$ of the patients persisted receiving the antiretroviral regularly, while $56(44.1 \%)$ of the patients had no monthly acquired the medicines or acquired intermittently. The data point out to the importance of the establishment of pharmaceutical care service for advising the HIV infected patients, which could improve the patient adhesion to the therapeutic schedule.

KEYWORDS: Aids; antiretroviral; dispensation. 


\section{INTRODUÇÃO}

A AIDS (Síndrome da Imunodeficiência Adquirida) é uma doença cuja história é um exemplo não só de investigação epidemiológica, mas também da preocupação político social, pois atinge milhares de pessoas em todo o mundo.

A epidemia de HIVIAIDS configura um dos mais sérios problemas contemporâneos de saúde pública, apresentando alto grau de morbi-mortalidade e perspectivas de um contínuo crescimento e propagação em todos os continentes (WHO, 2006). Apesar dos avanços, as possibilidades de controle e cura dessa doença ainda parecem remotas e mantém-se em expansão (ACÚRCIO; GUIMARÃES, 1999), apesar do desenvolvimento de novas terapias.

A AIDS é uma doença do sistema imunológico causada pelo HIV (Vírus da Imunodeficiência Humana), que apresenta uma ampla variedade de formas clínicas, desde assintomática até formas plenamente manifestas e que evoluem para morte. As manifestações clínicas decorrem conjuntamente da infecção pelo vírus HIV e outros patógenos que se instalam em decorrência das alterações imunológicas provocadas pela infecção primária. Dessa forma esta doença pode se apresentar de forma isolada ou associada a outras enfermidades, demonstrando assim a gravidade da síndrome, o que constitui um grave problema de saúde pública mundial (WHO, 2006).

De acordo com a Organização Mundial da Saúde (OMS) em 2006, das 39,5 milhões de pessoas vivendo com AIDS/HIV no mundo, houve 2,9 milhões de mortes causadas pela AIDS e o número de novas infecções foi de 4,3 milhões. (WHO, 2006). No Brasil, o número de pessoas vivendo com AIDS era de 620 mil (2005), representando 1/3 do número das pessoas que vivem com AIDS na América Latina (WHO, 2006).

O Brasil já conseguiu estabilizar a epidemia da AIDS, com uma taxa de prevalência menor do que $1 \%$. A partir de 1997 os pacientes tiveram um aumento de sobrevida em virtude do acesso a terapia anti-retroviral, através do programa de distribuição gratuita pelo Sistema Único de Saúde (SUS). Mas existe uma preocupação quanto ao futuro da política brasileira de acesso universal ao medicamento, pois os recursos são limitados e o número de doentes com AIDS só tendem a aumentar no Brasil, pois a doença tornou-se de caráter crônico, requerendo tratamento indefinidamente (BRASIL, 2005).

Como o vírus HIV compartilha muito dos processos metabólicos da célula hospedeira, é difícil encontrar fármacos que sejam seletivos para o patógeno. Todavia existem algumas enzimas que são específicas do vírus e que podem constituir alvos potenciais para fármacos. Os agentes antivirais atualmente disponíveis são em sua maioria eficazes apenas durante a replicação do vírus (RANG, 2003).

Os fármacos anti - HIV existente hoje, constituem a duas classes principais: os inibidores da transcriptase reversa e os inibidores da protease. Cada classe apresenta um mecanismo de ação diferente e são utilizados em combinações na terapia da AIDS. São eles:

- inibidores da transcriptase reversa, que são subdivididos em inibidores nucleosídios da transcriptase reversa : zidovudina (AZT), abacavir ( $A B C)$, lamivudina (3TC), didanosina (ddl), zalcitabina (ddC), estavudina (d4T); os inibidores não-nucleosídios da transcriptase reversa : efavirenz (EFZ) e nevirapina (NVP) e o inibidor de transcriptase reversa análogo de nucleotídeo: tenofovir (TDF).

- inibidores da protease: saquinavir (SQV), nelfinavir (NFV), indinavir (IDV), ritonavir (RTV) amprenavir (AMP), atazanavir (ATV) e lopinavir (LPV) ( RANG, 2003).

O uso da terapia de combinação modificou o prognóstico da AIDS, ela é conhecida como terapia antiretroviral altamente ativa (HAART), e esta combinação envolve dois inibidores nucleosídeos da transcriptase reversa com um inibidor não-nucleosídeo da transcriptase reversa ou um ou dois inibidores da protease (RANG, 2003).

Nos últimos cinco anos, várias drogas anti-retrovirais tem mostrado grande eficácia na inibição do ciclo de reprodução viral, reduzindo significativamente a gravidade das manifestações clínicas da doença. Informações de estudos realizados em vários países, incluindo o Brasil, efetuados em âmbito clínico, multi-institucional ou com dados de base populacional indicam uma diminuição importante em vários indicadores de morbidade e mortalidade de pacientes com HIVIAIDS, após a introdução da terapia anti-retroviral combinada incorporando medicamentos inibidores de protease (VITÓRIA, 1998). Tais observações coincidem com aumentos relevantes na sobrevida e melhora da qualidade de vida dessas pessoas. Apesar da alta eficácia da terapia anti-retroviral combinada, a efetividade tem sido prejudicada em virtude das dificuldades que os pacientes enfrentam para cumprir o tratamento corretamente. Aumentar a aderência dos pacientes ao tratamento anti-retroviral constitui uma questão de primeira ordem no combate à evolução da epidemia (CARVALHO, et al. 2003 ; FIGUEREDO, 2001).

No caso específico da terapia anti-retroviral, os regimes terapêuticos atualmente recomendados apresentam-se ainda com alto grau de complexidade ( 2 a 3 doses ao dia, podendo interferir no regime alimentar), 
com um grande número de comprimidos ou cápsulas e utilizados por tempo indeterminado, o que dificultam sobremaneira a adesão terapêutica a longo prazo (CARVALHO, et al. 2003); estudos mostram que a adesão decresce com o aumento do número de pílulas,cápsulas ou comprimidos que o paciente tem que tomar por dia ( CHESNEY et al., 2000). Uma taxa de aderência menor que 80\% está associada a viremia detectável na maioria dos pacientes (CHESNEY, 2000).

Entretanto, a não adesão ao tratamento anti-retroviral está diretamente relacionada com o desenvolvimento de resistência viral, com conseqüente falência terapêutica (MURPHY, et al., 2000; KASTRISSIOS, et al., 1998) e surgimento de cepas virais multiresistentes (VITÓRIA, 1998). Assim, todo o imenso investimento na pesquisa e desenvolvimento de drogas anti-retrovirais, bem como na tecnologia necessária para o acompanhamento da eficácia desta terapia poderão ser perdidos, se não forem elaboradas e padronizadas algumas estratégias que visam aumentar a adesão do paciente portador de HIVIAIDS ao tratamento (VITÓRIA, 1998; BRASIL, 2005).

O tratamento com anti-retrovirais é considerado um compromisso para a vida toda. Isso porque os esquemas considerados como os mais bem sucedidos são os que reduzem a carga viral do HIV a níveis baixos. Se houver a suspensão do tratamento, este controle fica comprometido, devido o aumento da carga viral e a diminuição da contagem de células CD4, causando grande risco a saúde do paciente (CECCATO, 2004).

Com a introdução da terapia anti-retroviral combinada como padrão de qualidade no tratamento das pessoas vivendo com HIVIAIDS, a adesão do paciente a esquemas terapêuticos com múltiplas drogas tornou-se fundamental no manejo clínico dessa doença. Dados de alguns estudos mostram que a taxa de aderência ao tratamento está entre 70 a 80\% (HAUBRICH, et al., 1999; HOWARD, et al., 2002).

A não adesão aos novos medicamentos anti-retrovirais, em geral, e inibidores de proteases, em particular tem sido considerada como um perigo para a efetividade do tratamento, na dimensão individual, e para a disseminação de vírus-resistência, em nível coletivo. Os estudos que têm avaliado fatores de não adesão à terapia anti-retroviral apontaram fatores étnicos, uso de drogas, efeitos colaterais, freqüências de doses e fatores socioeconômicos como relevantes. A falta de adesão total ao tratamento pode ser atribuída ainda a problemas psicológicos, culturais e sociais (CHESNEY, 2000; MALTA, et al., 2005; BRITO; SZWARCWALD; CASTILHO, 2006).

No Brasil o medicamento anti-retroviral é de fácil acesso, visto que o Ministério da Saúde mantém um programa nacional de distribuição gratuita destes medicamentos, através dos diversos Serviços de Atendimento Especializado (SAE) existentes em todos os estados brasileiros. Parte do grande sucesso do programa de AIDS no Brasil, deve-se a garantia de acesso universal aos medicamentos (NEMES, et al., 2004), estudos mostram que a disponibilidade de ARV é alta e homogênea, não faltam medicamentos em 95,5\% dos serviços (MELCHIOR et al., 2006). Mas além do acesso universal aos medicamentos, os indivíduos com AIDS também necessitam de qualidade no cuidado prestado pelos serviços, para que a adesão ao tratamento aumente cada vez mais.

Vários indicadores evidenciam o efeito positivo dessa política adotada no país, como uma redução da mortalidade $(50,0 \%)$, diminuição das internações hospitalares $(80,0 \%)$, redução da incidência de infecções oportunistas, e da transmissão vertical, dentre outros (WHO, 2006; BRITO; SZWARCWALD; CASTILHO; 2006).

Face a gravidade desta doença, e a dificuldade de adesão a terapia medicamentosa, já avaliada em vários serviços no Brasil, considerou-se importante a realização de um estudo preliminar da dispensação de medicamentos anti-retrovirais no serviço de farmácia do Hospital das Clínicas/UFG, cuja finalidade principal era obter dados do atendimento e conhecer melhor esta atividade, para desenvolver posteriormente um projeto de atenção farmacêutica a estes pacientes.

\section{MATERIAL E METODOLOGIA}

Tratou-se de uma pesquisa descritiva com levantamento de dados quantitativos, baseado no referencial, fichas de cadastro e de atendimento ambulatorial usadas durante a dispensação de medicamentos anti-retrovirais aos pacientes portadores de HIV cadastrados no SAE HC/UFG.

A coleta dos dados foi de forma manual nas fichas de cadastros e de atendimento ambulatorial. Após a coleta dos dados gerais, como: número de pacientes cadastrados, sexo, idade, esquemas terapêuticos, droga mais usada, intervalo de tempo da retirada do medicamento pelo paciente, tempo de uso, abandono de tratamento etc., estes foram analisados e submetidos análise percentual.

\section{População}

A amostra foi constituída por dados retirados de fichas de todos os pacientes cadastrados no SAE HC/UFG. Foram excluídos aqueles que pacientes com cadastro incompleto. O período de coleta dos dados aconteceu a partir do momento que o paciente passou a receber os medicamentos na seção de farmácia do HC/UFG até o mês de fevereiro/2007. 
A amostra consistiu em 127 fichas de pacientes de um total de 158 cadastrados nesse SAE. Dos 158 pacientes cadastrados no SAE HC/UFG foram excluídos 31 pacientes desta análise devido ao cadastro incompleto, resultando em uma amostra final de 127 pacientes.

\section{Questão ética}

Não houve identificação nominal, nem risco moral para os pacientes por se tratar apenas de dados estatísticos. Este trabalho foi submetido ao Comitê de Ética em Pesquisa do HC/UFG, e recebeu o seguinte número de protocolo: 017/07.

\section{RESULTADOS E DISCUSSÃO}

As características sociodemográfica da população estudada mostram predominância do sexo masculino com $91(71,6 \%)$ e $36(28,4 \%)$ do sexo feminino. A faixa etária mais prevalente do total de pacientes foi a de $31-40$ anos, o que coincidiu com a mesma do sexo masculino, mas em relação às mulheres a faixa etária mais prevalente foi a de 41- 50 anos (Tabela 1).

Esse resultado é condizente com a literatura brasileira no que diz respeito a pacientes portadores de HIVIAIDS (NEMES et al., 2000) e com dados do ministério da saúde (BRASIL, 2006).

Tabela1: Distribuição de freqüência da população estudada de acordo com a faixa etária ( n=127).

\begin{tabular}{c|ccc}
\hline Faixa Etária & Masculino & Feminino & Total \\
& & & \\
\hline $20-30$ anos & 24 & 7 & 31 \\
& $26,4 \%$ & $19,4 \%$ & $24,4 \%$ \\
\hline $31-40$ anos & 33 & 10 & 43 \\
& $36,2 \%$ & $27,8 \%$ & $33,8 \%$ \\
\hline $41-50$ anos & 22 & 13 & 35 \\
& $24,2 \%$ & $36,2 \%$ & $27,6 \%$ \\
\hline $51-60$ anos & 8 & 5 & 13 \\
& $8,8 \%$ & $13,9 \%$ & $10,3 \%$ \\
\hline$>60$ anos & 4 & 1 & 5 \\
& $4,4 \%$ & $2,7 \%$ & $3,9 \%$ \\
\hline
\end{tabular}

Em relação a escolaridade o nível de maior percentual foi o fundamental com 51,2\% seguido do nível médio com $33,8 \%$, nível superior completo ou incompleto $12,6 \%$ e $2,4 \%$ dos pacientes eram analfabetos; conforme demonstrado na tabela 2.

Tabela 2: Distribuição da população estudada de acordo com a escolaridade ( $n=127$ )

\begin{tabular}{l|ll}
\hline Escolaridade & $\mathbf{N}^{\circ}$ & $\%$ \\
\hline Analfabeto & 3 & 2,4 \\
Nível Fundamental & 65 & 51,2 \\
Nível Médio & 43 & 33,8 \\
Nível Superior Completo e Incompleto & 16 & 12,6 \\
\hline
\end{tabular}

Quanto a distribuição dos pacientes por nível de escolaridade, o padrão encontrado no estudo mostra-se semelhante ao geral para o Brasil, que se caracteriza por uma freqüência maior entre pessoas que tem baixo grau de instrução (BRASIL, 2006)

Em relação a dispensação mensal dos medicamentos na farmácia, $71(55,9 \%)$ pacientes retiravam os antiretrovirais regularmente, enquanto que $56(44,1 \%)$ em algum momento após o início do tratamento não retiraram os medicamentos mensalmente ou retiravam totalmente irregular, conforme mostra a figura 1 . Estes achados mostram uma provável existência de não adesão ao tratamento por um número significante de pacientes. Estudos realizados em Minas Gerais e no Rio Grande do Norte, onde avaliaram a adesão a terapia, a partir da dispensação pela farmácia, verificou-se as seguintes taxas de aderência, $76,5 \%$ e $64,1 \%$ respectivamente 
(LIGNANI, GRECO, CARNEIRO, 2001; BRITO, SZWARCWALD, CASTILHO, 2006). Com base na dispensação, podemos inferir que a adesão observada neste estudo foi menor, que as apresentadas em outros estudos.

Importante ressaltar que a não dispensação dos medicamentos não foi em nenhum momento pela falta do medicamento na farmácia ou falha na dispensação, mas sim pelo não comparecimento do paciente ou responsável ao serviço de farmácia para receber os anti-retrovirais mensalmente.

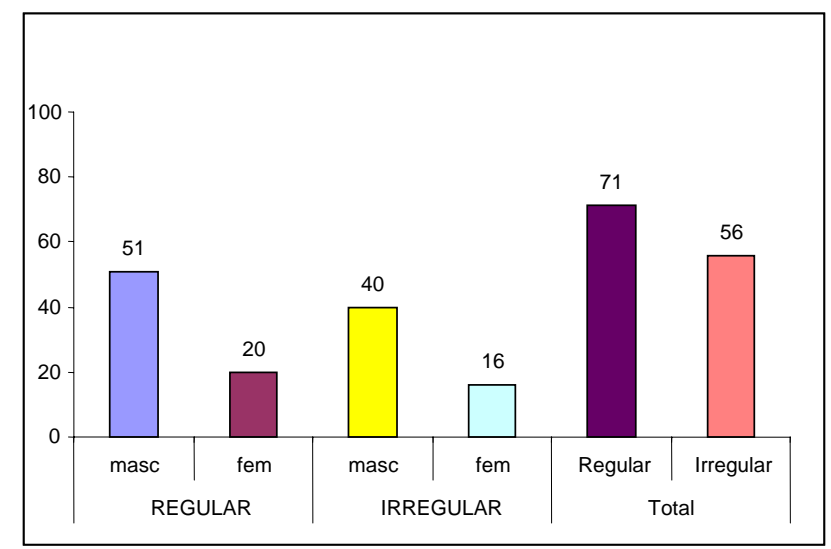

Figura1: Comparecimento mensal dos pacientes para a retirada dos medicamentos anti-retrovirais no SAE HC/UFG.

Mesmo sabendo que a adesão ao tratamento não se refere apenas a aquisição do medicamento por parte do paciente, consideramos que o não comparecimento á farmácia, com uma conseqüente não retirada dos antiretrovirais, seja um fator influente e significativo na análise da aderência por parte destes pacientes ao tratamento. No caso dos anti-retrovirais este fator se torna mais importante, visto que são medicamentos de custo elevado e de difícil aquisição no comércio. Então considerando apenas a dispensação dos medicamentos no serviço de farmácia do HC/UFG, verificou-se que cerca $50 \%$ dos pacientes não aderiam ao tratamento, pois nos meses em que não havia a retirada do medicamento, duas hipóteses poderiam acontecer: o paciente fazia o uso inadequado ou deixavam de tomar em algum momento, visto que estes medicamentos são dispensados de forma individual, e apenas para um período de 30 dias.

Em alguns estudos realizados no Brasil observou-se uma taxa de adesão bem variável, conforme mostrado nestes trabalhos: 69\% em 27 unidades do Estado de São Paulo (NEMES et al., 2000), 85,8 \% em hospital dia de Brasília (CARVALHO et al., 2003) e 49\% em SAE de Pelotas/RS (SILVEIRA et al., 2003). A supressão viral, a redução das taxas de resistências e melhora na sobrevida tem sido correlacionado com altas taxas de aderência a terapia anti-retroviral (OARAC,2006).

Observou-se também que a regularidade de retirada do medicamento na farmácia entre as mulheres $(55,5 \%)$ e entre os homens (56 \%) foi equivalente, consequentemente a irregularidade também.

Em alguns estudos a variável sexo não mostrou associação estatística com adesão, embora tenha sido encontrada uma menor aderência entre as mulheres, provavelmente devido ao tamanho da populaçao avaliada (CARVALHO et al.,2003).

No que se refere ao número de pacientes que mudaram de tratamento, 13,3\% $(n=17)$ apresentaram alteração no esquema terapêutico. Pacientes que fizeram mudança no tratamento, e a relação entre regularidade de retirada dos medicamentos na farmácia, são demonstrados na tabela 3, sendo que estas aconteceram principalmente nos pacientes que recebiam de forma irregular os medicamentos.

Tabela 3: A porcentagem dos pacientes que mudaram a terapia medicamentosa e a relação com retirada dos medicamentos na farmácia

\begin{tabular}{l|ccc}
\hline Retirada & $\mathbf{N}^{\circ}$ Pacientes & Mudança & $\%$ \\
\hline Regular & 71 & 5 & 7,0 \\
Irregular & 56 & 12 & 21,4 \\
\hline Total & 127 & 17 & 13,3 \\
\hline
\end{tabular}


Em relação aos esquemas terapêuticos, foi observado o uso de dez tipos de esquemas diferentes, sendo o predominante zidovudina + lamivudina + efavirenz (AZT+3TC+EFZ) representando 75,6\% (96 pacientes) do total. Os outros esquemas terapêuticos usados pelos pacientes estão demonstrados na tabela 4.

Tabela 4: Demonstração e distribuição dos esquemas terapêuticos usados pelos pacientes do SAE HC/UFG, quanto ao sexo, percentual e total.

\begin{tabular}{|c|c|c|c|c|c|c|}
\hline ESQUEMA TERAPÊUTICO & MASC & $\%$ & FEM & $\%$ & TOTAL & $\%$ \\
\hline $\mathrm{AZT}+3 \mathrm{TC}+\mathrm{EFZ}$ & 69 & 54,3 & 27 & 21,3 & 96 & 75,6 \\
\hline$A Z T+3 T C+N V P$ & 5 & 3,9 & 3 & 2,4 & 8 & 6,3 \\
\hline AZT + 3TC+ ATV 150+ RTV & 5 & 3,9 & 2 & 1,6 & 7 & 5,5 \\
\hline AZT + 3TC+ ATV 200 & 3 & 2,4 & 1 & 0,8 & 4 & 3,1 \\
\hline $\mathrm{AZT}+3 \mathrm{TC}+\mathrm{LPVr}$ & 3 & 2,4 & 1 & 0,8 & 4 & 3,1 \\
\hline AZT + 3TC+ ATV 150+ RTV +TDF & 2 & 1,6 & 1 & 0,8 & 3 & 2,4 \\
\hline$A B C+3 T C+A T V 200+R T V+T D F$ & 1 & 0,8 & 1 & 0,8 & 2 & 1,6 \\
\hline ATV + RTV + EFZ + TDF & 1 & 0,8 & 0 & 0 & 1 & 0,8 \\
\hline $\mathrm{DDI}+3 \mathrm{TC}+\mathrm{LPVr}$ & 1 & 0,8 & 0 & 0 & 1 & 0,8 \\
\hline$A Z T+3 T C+L P V r+T D F$ & 1 & 0,8 & 0 & 0 & 1 & 0,8 \\
\hline
\end{tabular}

ABC = Abacavir; AZT= Zidovudina; ATV = Atazanavir; DDI = Didanosina; EFZ = Efavirenz; d4T = Estavudina; 3TC = Lamivudina; LPVr = Lopinavir/Ritonavir; NVP = Nevirapina; RTV = Ritonavir; TDF = Tenofovir

Observou-se que os esquemas mais usados compreendiam o de três fármacos, correspondendo a 85\% do total dos pacientes analisados.

Dos pacientes com esquema tríplice ou com mais combinações, 18,1 \% ( $n=23$ ) fizeram uso de inibidor de protease. Dentre os 277 medicamentos prescritos a Zidovudina associada à Lamivudina apresentou-se como o medicamento mais prescrito, seguido pelo Efavirenz e Atazanavir, conforme mostra a figura 2.

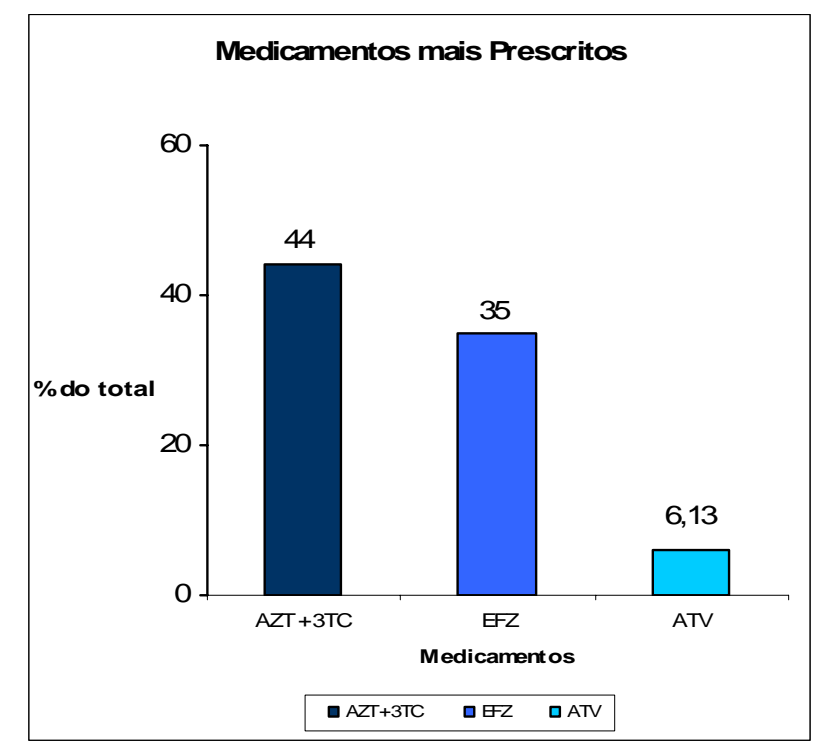

Figura 2: Medicamentos mais prescritos para os pacientes atendidos no SAE HC/UFG $(A Z T+3 T C=$ zidovudina +lamivudina; EFZ = efavirenz e ATV = atazanavir).

De acordo com o Consenso Brasileiro, o tratamento anti-retroviral é indicado para todos os pacientes infectados pelo HIV, sintomático ou assintomático, que apresentam contagem de linfócitos T-CD4+ abaixo de $200 / \mathrm{mm}^{3}$, o início da terapia deve ser considerado conforme a evolução dos parâmetros imunológicos, virológicos e outras características do paciente. Os esquemas preferenciais recomendados são os constituídos por dois inibidores de transcriptase reversa análogo de nucleosídeos (ITRN - zidovudina, lamivudina) e um inibidor de transcriptase reversa não-análogo de nucleosídeos (ITRNN - efavirenz); o uso de inibidor de protease (IP) passou 
a ter uma indicação mais restrita, sendo recomendado apenas para pacientes com imunodeficiência grave e como resgate terapêutico (BRASIL, 2002). Neste estudo, a maioria dos pacientes usam o esquema terapêutico com dois ITRN e um ITRNN e 14,9\% dos pacientes já iniciaram o tratamento incluindo o IP ao esquema.

Outros dados mostram também, que o número de fármacos no esquema terapêutico não interferiu na regularidade de recebimento dos medicamentos, pois o número de pacientes que mais recebiam de forma irregular os anti-retrovirais no serviço de farmácia do HC/UFG foram aqueles que usam somente três fármacos ou dois medicamentos, pois um deles é uma associação. Os pacientes que recebiam de forma mais irregular usam os seguintes esquemas: AZT + 3TC + EFZ ( $n=34) ; A Z T+3 T C+N V P(n=4) ; A Z T+3 T C+A T V 200(n=4)$, este resultado era esperado para o esquema $A Z T+3 T C+E F Z$, pois é o mais usado entre a população estudada.

Quando se trata de adesão ao tratamento, o número de medicamentos usado pelos pacientes, corresponde um fator importante, conforme mostra o estudo realizado no HC-UNICAMP, cuja adesão é menor no grupo que tomavam mais dose/dia e mais medicamentos (FIGUEIREDO, 2001). Em um estudo no SAE de Pelotas/RS, mostra que pacientes com prescrição de três a quatro doses diárias a possibilidade de adesão diminui em 53\%, quando comparado a esquemas com regime de duas doses, e quando foi prescrito regime de cinco a seis doses a probabilidade de adesão foi 76\% menor (SILVEIRA et al., 2003).

Neste estudo, verificou-se também que houve um crescimento no número de pacientes que iniciaram tratamento com anti-retroviral, no SAE HC/UFG, sendo que o maior aumento aconteceu nos últimos três anos, representando um aumento de $71,6 \%$, conforme mostra a figura 3.

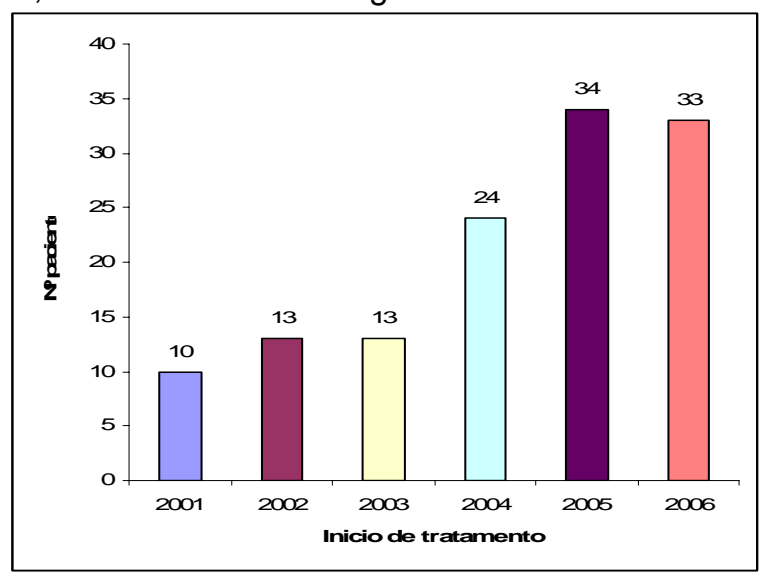

Figura 3 : Número de pacientes que iniciaram tratamento no SAE HC/UFG, no período de 2001 a 2006.

Neste trabalho, índices relacionados ao comparecimento dos pacientes ao serviço de farmácia foram utilizados para avaliar aspectos em relação ao tratamento. Trata-se de um método indireto, fácil e útil para avaliar interrupção de tratamento, mas implica a subestimação do nível de adesão, pois uma das limitações, é que esta medida não permite afirmar se os medicamentos foram tomados na quantidade prescrita e nos horários definidos, mas é possível considerar que um paciente que segue as recomendações médicas e comparece regularmente a unidade de assistência farmacêutica está seguindo a terapia medicamentosa de forma adequada.

\section{CONCLUSÃO}

Os dados obtidos neste trabalho apontam para a existência de uma provável não adesão do tratamento, de cerca de $50 \%$ dos pacientes atendidos pelo serviço de farmácia do HC/UFG, pois em algum momento do seu tratamento, estes deixaram de comparecer para a retirada dos medicamentos anti-retrovirais, ou compareciam de forma totalmente irregular. E como são medicamentos de difícil aquisição e preço elevado, acredita-se que os pacientes que não comparecem a farmácia, não adquirem de outra forma, portanto estão deixando de seguir corretamente o esquema farmacoterapêutico.

Acredita-se que esta falha no recebimento deva-se a diversos fatores, como: baixa escolaridade, orientação insuficiente, falta de compreensão, conhecimento inadequado, estilo de vida, crenças, fatores sócio econômico, etc. Portanto é de fundamental importância o papel da comunicação do profissional de saúde com o paciente na implementação de estratégias para melhorar a adesão ao tratamento. A participação do Farmacêutico no trabalho em equipe é essencial, para melhor compreensão do tratamento por parte dos pacientes, conforme mostrou um estudo realizado em Belo Horizonte em 2001-2002 (CECCATO et al., 2004). Portanto o enfoque multidisciplinar no atendimento aos pacientes poderá ser a alternativa mais eficaz para que os pacientes atendidos no SAE HC/UFG tenham uma melhor assistência e uma melhor adesão ao tratamento. 
Os resultados obtidos serão úteis, pois mostram com clareza pontos importantes, para a construção de um projeto de atenção farmacêutica que o Serviço de Farmácia do HC/UFG, busca desenvolver para melhor atender e orientar os pacientes portadores de HIVIAIDS.

\section{REFERÊNCIAS BIBLIOGRÁFICAS}

ACURCIO, F. A.; GUIMARÃES, M.D. C. Utilização de medicamentos por indivíduos HIV positivos: abordagem qualitativa. Rev. Saúde Pública,São Paulo 33 (1):73-84, 1999.

BRASIL. Ministério da Saúde. Secretaria de Vigilância em Saúde. Programa Nacional de DST e Aids. O Remédio via Justiça. Secretaria da Vigilância em Saúde, Programa Nacional de DST e Aids. - Brasília: 2005.

BRASIL. Ministério da Saúde. Coordenação Nacional DST/Aids. Recomendações para terapia antiretroviral em adultos e adolescentes infectados pelo HIV -2002/2003 - Brasília: 2002. Disponível em: http: www.aids.gov.br/final/tratamento/adulto [capturado em 25 maio 2007].

BRASIL. Ministério da Saúde. Boletim epidemiológico Aids, ano III, nº1. 2006. Disponível em: http: www.aids.gov.br/final/data/documentos [capturado em 25 maio 2007].

BRITO, A.M SZWARCWALD C. L; CASTILHO, E.A. Fatores associados à interrupção de tratamento anti-retroviral em adultos com AIDS. Rev. Assoc. Med. Bras, São Paulo, v.52, n.2, 2006.

CARVALHO, C.V. et al. Determinantes da aderência à terapia anti-retroviral combinada em Brasília, Distrito Federal, Brasil, 1999-2000. Cad. Saúde Pública, Rio de Janeiro, v.19, n.2, 2003.

CECCATO, M. G. B. et al . Compreensão de informações relativas ao tratamento anti-retroviral entre indivíduos infectados pelo HIV. Cad. Saúde Pública, Rio de Janeiro, v. 20, n. 5, 2004.

CHESNEY, M. et al. Self-reported adherence to antiretroviral medications among participants in HIV clinical trials: the AACTG adherence instruments. AIDS Care, v.12, n.3, 2000.

CHESNEY, M. A. Factors affecting adherence to antiretroviral therapy. Clin Infect Dis. v.30 suppl, n.2, 2000.

FIGUEREDO. R. M. et al. Adesão de pacientes com AIDS ao tratamento com antiretovirais: Dificuldades relatadas e proposição de medidas atenuantes em um hospital escola. Rev Latino-am Enfermagem, v.9, n.4, p.50-5, 2001.

GARCIA, R.; SCHOOLEY, R.T.; BADARÓ, R. Na adherence trilogy is essential for long-term HAART sucess. The Brazilian Journal of Infectious Diseases, v.7, n.5, p. 307-314, 2003.

HAUBRICH, R. et al. The value of patient reported adherence to therapy in predicting virologic and immunologic response. AIDS, v.13, p.1099-107, 1999.

HOWARD, A.A. et al. A prospective study of adherence and viral load in a large multi-center cohort of HIV infected women. AIDS, v.16, p.2175-182, 2002.

JÚNIOR , L. L.; GRECO, D. B.; CARNEIRO ; M. Avaliação da aderência aos anti-retrovirais em pacientes com infecção pelo HIVIAids. Rev. Saúde Pública, São Paulo v. 35, n. 6, 2001.

KASTRISSIOS, H. et al. The extent of non-adherence in a large AIDS clinical trial using plasma dideoxynucleoside concentrations as a maker. AIDS, v.12, p.2305-11, 1998.

MALTA, M. et al. Adherence to antiretroviral therapy: a qualitative study with physicians from Rio de Janeiro, Brazil. Cad. Saúde Pública, Rio de Janeiro, v.21, n.5, 2005.

MELCHIOR, R. et al. Avaliação da estrutura organizacional da assistência ambulatorial em HIVIAids no Brasil. Rev. Saúde Pública, São Paulo v.40, n.1, 2006. 
MURPHY, D. et al. Barriers to antiretroviral adherence among HIV infected adults. AIDS, Patient Care. v.14, p.47$58,2000$.

NEMES, M.I.B. et al. Avaliação da qualidade da assistência no programa de AIDS: questões para a investigação em serviços de saúde no Brasil. Cad. Saúde Pública, Rio de Janeiro, v.20 sup, n.2, p. 5310-5321, 2004.

NEMES, M.I.B. et al. Avaliação da aderência ao tratamento por anti-retrovirais em usuários de ambulatórios do sistema público de assistência à Aids no estado de São Paulo. Coordenação Nacional de DST/Aids. Brasília: Ministério da Saúde. 2000. www.aids.gov.br/data/documents [capturado em 25, maio 2007].

OARAC-Office Aids Research Advisory Council. Guidelines for the use of antiretroviral agents in HIV-1 infected adults and adolescents, 2006. Disponível em: http://aidsinfo.nih.gov [capturado em 25, maio 2007].

SILVEIRA, V.L. et al. Characteristics of HIV antiretroviral regimen and treatment adherence. The Brazilian Journal of Infectious Diseases, v.7, n.3, p. 194-201, 2003.

RANG, h. P. et al. Farmacologia. 5 ed. Rio de Janeiro: Elsevier, 2003, 904 p.

VITÓRIA, M.A.A. Conceitos e recomendações básicas para melhorar a adesão ao tratamento antiretroviral. Coordenação Nacional DST/AIDS. Brasília: Ministério da Saúde, 1998.

WHO, Dados UNAIDS - Joint United Nations Programme on HIVIAIDS. www.unaids.org , 2006. 